\title{
An EST-based approach for identifying genes expressed in the intestine and gills of pre-smolt Atlantic salmon (Salmo salar) Heidi Hagen-Larsen ${ }^{1}$, Jon K Laerdahl ${ }^{2}$, Frank Panitz ${ }^{3}$, Alexei Adzhubei ${ }^{2}$ and Bjørn Høyheim*1,4
}

Address: ${ }^{1}$ Norwegian School of Veterinary Science, Department of Basic Sciences and Aquatic Medicine. PO Box 8146 Dep., NO-0033 Oslo, Norway, ${ }^{2}$ Biotechnology Centre of Oslo (BIO), University of Oslo, PO Box 1125 Blindern, 0317 Oslo, Norway, ${ }^{3}$ Danish Institute of Agricultural Sciences, Department of Animal Breeding and Genetics, PO Box 50, DK-8830 Tjele, Denmark and ${ }^{4}$ Rikshospitalet University Hospital, Department of Dermatology, NO-0027 Oslo, Norway

Email: Heidi Hagen-Larsen - heidi.hagen_larsen@veths.no; Jon K Laerdahl - j.k.lardahl@medisin.uio.no; Frank Panitz - frank.panitz@agrsci.dk; Alexei Adzhubei - a.a.adjoubei@biotek.uio.no; Bjørn Høyheim* - Bjorn.Hoyheim@veths.no

* Corresponding author

Published: 0 I December 2005

BMC Genomics 2005, 6:17| doi:|0.1|86/|47|-2|64-6-|7|
Received: 15 June 2005

Accepted: 01 December 2005

This article is available from: http://www.biomedcentral.com/I47|-2/64/6/17|

(c) 2005 Hagen-Larsen et al; licensee BioMed Central Ltd.

This is an Open Access article distributed under the terms of the Creative Commons Attribution License (http://creativecommons.org/licenses/by/2.0), which permits unrestricted use, distribution, and reproduction in any medium, provided the original work is properly cited.

\begin{abstract}
Background: The Atlantic salmon is an important aquaculture species and a very interesting species biologically, since it spawns in fresh water and develops through several stages before becoming a smolt, the stage at which it migrates to the sea to feed. The dramatic change of habitat requires physiological, morphological and behavioural changes to prepare the salmon for its new environment. These changes are called the parr-smolt transformation or smoltification, and preadapt the salmon for survival and growth in the marine environment. The development of hypoosmotic regulatory ability plays an important part in facilitating the transition from rivers to the sea. The physiological mechanisms behind the developmental changes are largely unknown. An understanding of the transformation process will be vital to the future of the aquaculture industry. A knowledge of which genes are expressed prior to the smoltification process is an important basis for further studies.
\end{abstract}

Results: In all, 2974 unique sequences, consisting of 779 contigs and 2195 singlets, were generated for Atlantic salmon from two cDNA libraries constructed from the gills and the intestine, accession numbers [Genbank: CK877169-CK879929, CK8840I5-CK886537 and CNI8III2-CNI8I464]. Nearly $50 \%$ of the sequences were assigned putative functions because they showed similarity to known genes, mostly from other species, in one or more of the databases used. The Swiss-Prot database returned significant hits for 1005 sequences. These could be assigned predicted gene products, and 967 were annotated using Gene Ontology (GO) terms for molecular function, biological process and/or cellular component, employing an annotation transfer procedure.

Conclusion: This paper describes the construction of two cDNA libraries from pre-smolt Atlantic salmon (Salmo salar) and the subsequent EST sequencing, clustering and assigning of putative function to 1005 genes expressed in the gills and/or intestine. 
Table I: Fraction sizes and number of sequences for the gills and intestine cDNA libraries

\begin{tabular}{|c|c|c|c|c|c|}
\hline Library fraction & Mean insert size & Clones seq. ${ }^{c}$ & Good seq.c & Unique seq.c & Annotated seq.c \\
\hline Gills 5 & I,500 bp & & & & \\
\hline Gills 6 & $900 \mathrm{bp}$ & & & & \\
\hline Gills 7 & 700 bp & 4128 & 3015 & & \\
\hline Intestine 4 & $1,300 \mathrm{bp}$ & & & & \\
\hline Intestine 5 & $\mathrm{I}, 050 \mathrm{bp}$ & & & & \\
\hline Intestine 6 & $730 \mathrm{bp}$ & 4128 & 3247 & & \\
\hline Total & & & & 2974 & 1005 \\
\hline
\end{tabular}

\footnotetext{
a The numbers after the tissue type indicate when the cDNA was collected from the column, the lowest numbers being eluted first. The mean insert sizes are given in the second column

b Shown in base pairs

c Seq. is an abbreviation of sequence
}

\section{Background}

The number of known genes in Atlantic salmon (Salmo salar) is limited compared with the very large numbers known in mammals. However, the salmon is so important as an aquaculture species that a lot of effort has been put into acquiring knowledge of its genome compared to many of the other aquatic species. Although only 14 fulllength genes are listed for Atlantic salmon at NCBI [1], there are 111 457-nucleotide sequences (September 2005), many containing complete cDNA sequences. The genome and biology of the Atlantic salmon are complex and the genome has not been well characterised. The salmonidae species underwent a genome duplication event about 25-100 million years ago [2,3]. This event resulted in a tetraploid genome, but since then the salmon has been gradually returning to the diploid condition. This process is still continuing, and the salmon now has a partially tetraploid genome. Although many genes have been lost after the duplication event [4], the fact that regions of the genome are duplicated and almost identical does complicate the hunt for genes in this species. It means that there may be four almost identical copies of a gene in the genome, but it is an open question whether all four copies are active. Furthermore, the Atlantic salmon goes through a process known as smoltification as a step in its maturation. The salmon spends the first part of its life in fresh water before migrating to the ocean, where it lives until returning to rivers to spawn. Smoltification [5] involves synchronised morphological, physiological and behavioural changes that enable the young salmon (parr) to survive in the ocean and to grow and migrate normally. Since smoltification occurs while the fish are still in fresh water, they are pre-adapted to the marine environment. Given this enormous change in both fish biology and environment, it seems reasonable to suggest that the pre-smolt expresses a different set of genes from the smolt. It is therefore important to identify the genes that are expressed both before and after the smoltification process and at each of the different developmental stages in order to obtain a complete set of genes expressed in the salmon genome. One strategy for identifying the genes expressed in specific life stages and tissues is to use expressed sequence tags or ESTs. These are short stretches of single pass sequences obtained from sequencing cDNA $[6,7]$, and are widely used for gene discovery, mapping, polymorphism analysis, expression studies and gene prediction. Gene discovery methods using ESTs include hunting for new members of gene families in the same species (paralogues), for functionally equivalent genes in other species (orthologues), or even for alternatively spliced forms of known genes. ESTs are also used to predict or refine computational predictions of the location of genes in genomic DNA. Recently, a lot of effort has been put into acquiring ESTs from Atlantic salmon, and over 108,242 EST sequences (September 2005), mainly from smoltified fish, have been deposited in GenBank [811]The aim of the present study was to increase the number of ESTs available from pre-smolts in order to identify genes that are expressed in the early life stages of Atlantic salmon. We have focused on the gills and the intestine.

\section{Results}

One of the goals was to accumulate cDNA libraries with large insert sizes. The average insert size was determined from 192 clones from each library fraction after digesting the clones with EcoRI/Xhol. The approximate size distribution for the libraries is given in table 1 .

A total of 4128 clones from each library were sequenced from the 5'direction. After basecalling and trimming of vector, contaminants and poor quality sequences, the number of sequences was reduced to 3015 for the gills and 3247 for the intestine. The trimmed sequences were clustered and assembled. After the combined clustering of the gill and intestine sequences, a total of 2974 unique sequences were left, of which 2195 were singlets and 779 were consensus sequences from contigs. Of these, 1491 showed similarity to known sequences after they were run through a combined blastx and blastn pipeline. A sum- 
Table 2: Number of hits observed in the different databases

\begin{tabular}{|c|c|c|}
\hline Database & Hitsc & $\% d$ \\
\hline $\mathrm{PDB}^{\mathrm{a}}$ & 489 & 16 \\
\hline SwissProt ${ }^{b}$ & 1005 & 34 \\
\hline$n r^{b}$ & 1276 & 43 \\
\hline$n t^{b}$ & 961 & 32 \\
\hline no hits & |483 & 50 \\
\hline
\end{tabular}

a Threshold for a significant hit for blastx against PDB was defined as expectation value $<10^{-10}$

b Threshold for a significant hit for blastx against SwissProt and $\mathrm{nr}$, and blastn against $\mathrm{nr}(=\mathrm{nt})$ was defined as expectation value $<10-15$

c The number of significant hits observed in each databases

$d$ The number of significant hits observed given as a percentage

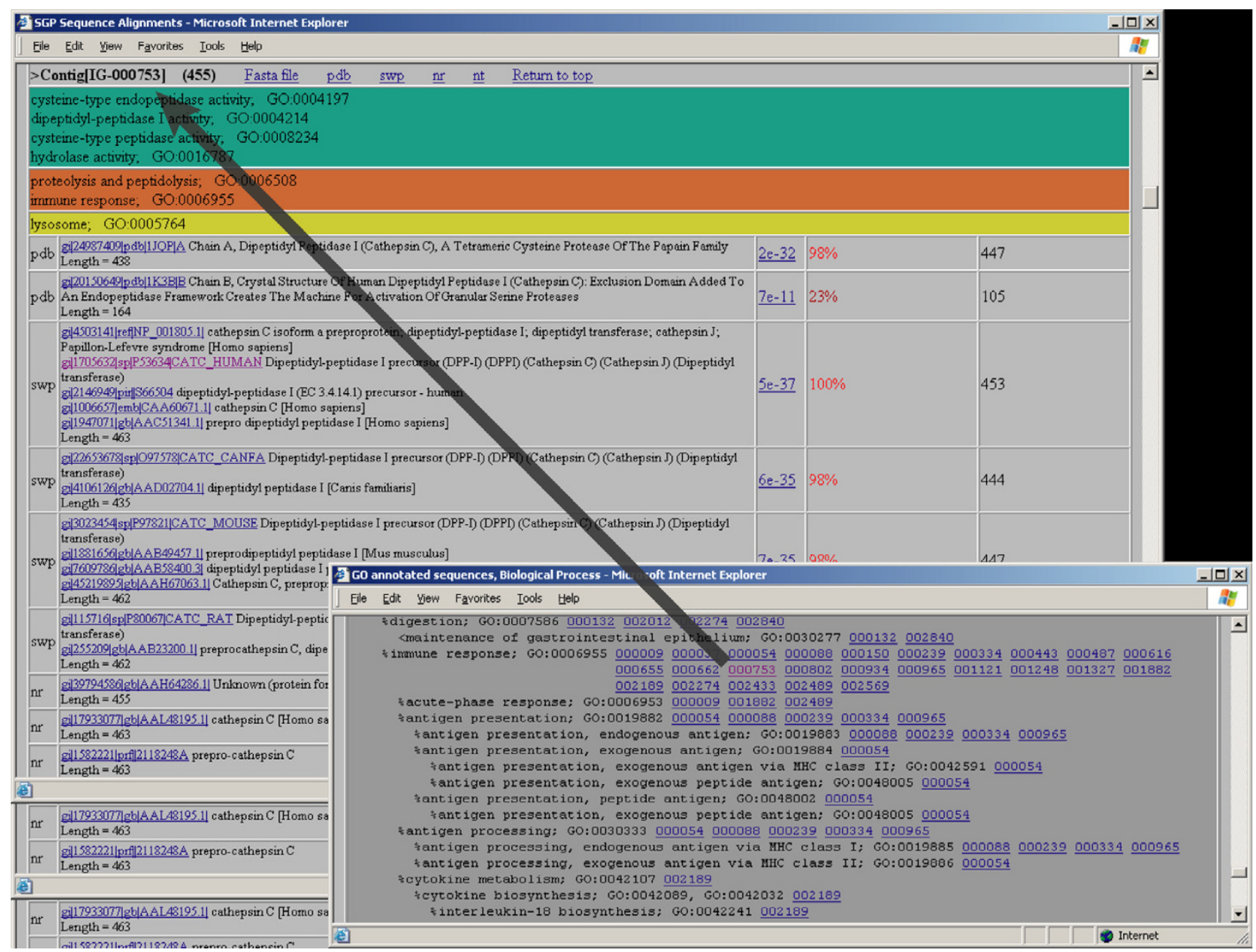

\section{Figure I}

Gene Ontology Annotation. The annotated sequences can be reached through three documents corresponding to molecular function, biological process, and cellular component GO terms. There are links to all results for a given GO term "immune response" is used in the figure - that can be followed to the sequence information and alignment summary for each sequence. The main tables give links to the fasta file containing the sequence itself and the BLAST results for each sequence. The most significant sequence alignment results are also given with various details such as expectation value and length of sequence alignment hit. See the web site for further details. 
Table 3: Gene Ontologya(Molecular function) of the sequences with a significant blastx hit in SwissProt

\begin{tabular}{|c|c|}
\hline Molecular Functionb & Nr of Hits \\
\hline \%antioxidant activity & 10 \\
\hline \%glutathione-disulfide reductase activity & 2 \\
\hline \%peroxidase activity & 5 \\
\hline \%binding & 505 \\
\hline \%antigen binding & I \\
\hline \%carbohydrate binding & 5 \\
\hline \%cofactor binding & I \\
\hline \%drug binding & I \\
\hline \%glycosaminoglycan binding & 12 \\
\hline \%isoprenoid binding & I \\
\hline \%lipid binding & 23 \\
\hline \%metal ion binding & 103 \\
\hline \%nucleic acid binding & 208 \\
\hline \%nucleotide binding & 132 \\
\hline \%peptide binding & 3 \\
\hline \%protein binding & 112 \\
\hline \%pyridoxal phosphate binding & I \\
\hline \%receptor binding & 17 \\
\hline \%selenium binding & I \\
\hline \%steroid binding & 2 \\
\hline \%tetrapyrrole binding & 2 \\
\hline \%vitamin binding & I \\
\hline \%catalytic activity & 358 \\
\hline \%helicase activity & 15 \\
\hline \%hydrolase activity & 162 \\
\hline \%integrase activity & I \\
\hline \%isomerase activity & 22 \\
\hline \%kinase activity & 28 \\
\hline \%ligase activity & 22 \\
\hline \%lyase activity & 11 \\
\hline \%oxidoreductase activity & 88 \\
\hline \%small protein activating enzyme activity & 2 \\
\hline \%small protein conjugating enzyme activity & 8 \\
\hline \%transferase activity & 59 \\
\hline \%transposase activity & 2 \\
\hline \%chaperone activity & 23 \\
\hline \%heat shock protein activity & 7 \\
\hline \%enzyme regulator activity & 30 \\
\hline \%caspase regulator activity & I \\
\hline \%enzyme activator activity & 10 \\
\hline \%enzyme inhibitor activity & 16 \\
\hline \%GTPase regulator activity & 9 \\
\hline$\%$ kinase regulator activity & 3 \\
\hline \%nitric-oxide synthase regulator activity & 1 \\
\hline \%molecular_function unknown & 29 \\
\hline \%motor activity & 11 \\
\hline \%microtubule motor activity & 2 \\
\hline \%signal transducer activity & 54 \\
\hline \%receptor activity & 26 \\
\hline \%receptor binding & 17 \\
\hline \%receptor signaling protein activity & 4 \\
\hline \%structural molecule activity & 160 \\
\hline \%extracellular matrix structural constituent & 2 \\
\hline \%structural constituent of bone & I \\
\hline$\%$ structural constituent of cytoskeleton & 10 \\
\hline \%structural constituent of eye lens & 2 \\
\hline \%structural constituent of muscle & 2 \\
\hline \%structural constituent of ribosome & 103 \\
\hline \%transcription regulator activity & 42 \\
\hline
\end{tabular}


Table 3: Gene Ontologya(Molecular function) of the sequences with a significant blastx hit in SwissProt (Continued)

\begin{tabular}{|c|c|}
\hline \%RNA polymerase I transcription factor activity & 1 \\
\hline \%RNA polymerase II transcription factor activity & 5 \\
\hline \%transcription cofactor activity & 11 \\
\hline \%transcription factor activity & 24 \\
\hline \%transcriptional repressor activity & 3 \\
\hline \%translation regulator activity & 21 \\
\hline \%translation factor activity, nucleic acid binding & 21 \\
\hline \%transporter activity & 156 \\
\hline \%amine/polyamine transporter activity & 2 \\
\hline \%auxiliary transport protein activity & $\mathrm{I}$ \\
\hline \%carbohydrate transporter activity & I \\
\hline \%carrier activity & 66 \\
\hline \%channel/pore class transporter activity & 8 \\
\hline \%drug transporter activity & $\mathrm{I}$ \\
\hline \%electron transporter activity & 34 \\
\hline \%intracellular transporter activity & 3 \\
\hline \%ion transporter activity & 58 \\
\hline \%lipid transporter activity & 5 \\
\hline \%neurotransmitter transporter activity & 2 \\
\hline \%organic acid transporter activity & 3 \\
\hline \%oxygen transporter activity & 6 \\
\hline \%peptide transporter activity & 2 \\
\hline \%protein transporter activity & 33 \\
\hline
\end{tabular}

a A selection of GO categories is presented. All annotations are accessible at http://www.salmongenome.no/

b Indented terms are 'children' of the above 'parent' term.

mary of the BLAST results is given in Table 2. All the sequence alignment results are accessible at the Salmon Genome Project website [11] in the libraries section (Data and Results > cDNA libraries > Gills, Intestine). All trimmed EST sequences have been submitted to GenBank. The accession numbers are [Genbank: CK877169CK879929, CK884015-CK886537 and CN181112CN181464]. The annotation of the clustered ESTs using GO terms is accessible through links at the Salmon Genome Project website [11] in the annotation section (Data and Results > Annotations > SGP Intestine-Gills). A shorter version is given in Table 3 for molecular function.

Table 3 shows that the sequences can be classified in 12 of the major categories for molecular function, the two largest groups being catalytic activity (26\%) and binding $(36 \%)$.

\section{Discussion}

The total number of gill and intestine clones available was 8256. Table 1 shows the sampled means of the insert sizes after fractionation, which varied between 700 and 1500 base pairs. cDNA cloning rarely produces full-length gene products but rather products containing varying proportions of the whole sequence. 5 'sequencing may overcome this problem since clustering can be used to cover larger parts of the transcripts. In this study, after trimming and quality control followed by clustering, approximately $65 \%$ of the trimmed sequences from both libraries ended up in contigs.
Sequence annotation was carried out using GO terms. A fairly strict criterion of E-value below $1.0 \times 10^{-15}$ for a significant hit was used both for Blastx run against the SwissProt and nr protein databases, and blastn against the nr nucleotide database. This rather low value was chosen to reduce the number of hits on very remote homologous genes with functions unrelated or only remotely related to the sequences in the current libraries. For blastx against the PDB database, the significance level chosen was an Evalue below $1.0 \times 10^{-10}$, and 489 or $16 \%$ of the sequences gave significant hits in this database. Almost all of these showed sequence similarity above 30\%, indicating that they might be suitable targets for homology modelling of protein $3 \mathrm{D}$ structure if the hit produces a biologically meaningful alignment.

Approximately one third of the sequences gave hits when blastx was run against the Swiss-Prot database, and were annotated by annotation transfer from GO-annotated UniProt sequences as described in section 2.6. Since we used a fairly strict criterion for a significant hit, we would argue that the derived GO annotation is meaningful for a large fraction of these 1005 sequences. We plan to use a more elaborate annotation pipeline for future work, including estimates of accuracy and sensitivity and making it possible to detect more remote homologous sequences, for example by profile-based sequence similarity searches. Annotating the sequences and collecting the links to all annotated sequences in tables that correspond to molecular function, biological process, and cellular component GO terms makes it possible in future studies 
to extract genes of interest merely by looking at the GO term tables from the links at the Salmon Genome Project website [11] in the Annotation section (Data and Results $>$ Annotations > SGP Intestine-Gills). Figure 1 illustrates this. A researcher interested in immunology would simply go to the biological process GO term table and search for 'immune response', and could then follow each of the 25 links to the sequences that appear to have a connection with immunology and their sequence alignment results. This will make it possible to find possibly relevant sequences much more directly, without having to browse through thousands of sequences and their associated sequence alignment data.

In the two libraries, we discovered several genes that may be involved in the smoltification process, genes involved in cell homeostasis and genes coding for hormones that may influence salmon maturation. Certain genes were of particular interest, for example the glucagon-family neuropeptide precursor, tyrosine 3/ tryptophan 5-monooxygenase activities and thyroid receptor interactin protein 6 (TRIP6). In addition, five different genes related to growth factor activity were also present: granulin, neuregulin 1, syntenin, Bmp2 and pleiotrohin 1. All of the above-mentioned genes are thought to play a part in the smoltification process in salmon, along with the osmoregulatory genes. However, it is possible that some of the more interesting genes involved in the smoltification process have not yet been annotated and characterised and are therefore listed as unknown. Approximately $50 \%$ of the sequences gave no significant hits in the blastx-blastn pipeline. A large proportion of these gave hits with higher E-values to more remote homologous sequences, but a significant number, at least 500-1000 sequences, are of completely unknown function at present. However, the 5' UTR of fish may differ significantly from that of mammals and terrestrial organisms. This means that the failure to match 500-1000 sequences does not confirm that the genes are unique to fish or markedly different from the coding region of transcripts of other organisms. A number of factors, including the 5'UTR, may help to explain why no similarity was found for these sequences, but the unusual biology of salmon and the fact that the sequences are from pre-smolt fish may be contributory factors. Presmolt salmon live in fresh water, and presumably different sets of genes are turned on and off in response to the two different habitats, the freshwater and the marine environment. In addition, the lack of similarity to already known sequences in other vertebrates could influence these results. Although large amounts of sequence data from other species have been generated, a large fraction of the genes in higher organisms is still uncharacterised. It therefore seems likely that the lack of similarity of these ESTs to known genes is partly explained by the fact that many genes are still uncharacterised.

\section{Conclusion}

This work has made available approximately 3000 sequences from pre-smolt salmon, one third of which have been annotated for function, biological process and cellular component. Several hundred sequences code for proteins that show enough sequence-similarity to proteins of known structure to be suitable candidates for homology modelling. The results of this study will be a valuable resource for future studies of Atlantic salmon biology since it is now possible to search for genes sequenced in salmon rather than having to use comparative data from other species.

\section{Methods}

\section{Construction of CDNA libraries}

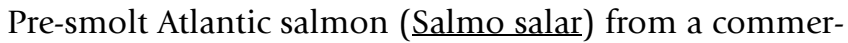
cial aquaculture-bred stock (National Norwegian Breeding Programme for salmon, Norway), weighing 50-100 g, were collected in late September for the construction of two cDNA libraries from the intestine and gills. The entire intestine and the whole of the gills were used to construct the libraries. Tissue samples of $400 \mathrm{mg}$ were used for total RNA extraction, which was performed using the guanidine thiocyanate-phenol-chloroform extraction method [12]. mRNA was further isolated using the Poly (A) Quick ${ }^{\circledast}$ mRNA isolation kit (Stratagene Cloning Systems, California, USA). Three $\mu \mathrm{g}$ of total RNA and $0.1 \mu \mathrm{g}$ of mRNA were run on an agarose gel to investigate the integrity/ quality of the samples. cDNA synthesis was performed using the pBluescript ${ }^{\circledR}$ II XR cDNA library construction kit (Stratagene Cloning Systems, California, USA), which generates directional cDNA libraries using an oligo $(\mathrm{dT})_{18}$ primer. For each library the cDNA was size fractionated using a gel filtration column, and three fractions containing most of the cDNA were ligated into the plasmid vector and subsequently transformed into XL10 Gold ultra competent cells.

\section{Pre-screening of the cDNA libraries}

Pre-screening was performed to separate abundant genes from rare ones. From each of the three cDNA fractions from both tissues, approximately 50000 colonies were plated out on agar plates (140 mm in diameter), with an average of 2000 colonies per plate. The colonies were transferred to $132 \mathrm{~mm}$ BIOTRANS nylon membranes, pore size 1.2 Micron, before hybridisation to total CDNA. Total cDNA from the intestine was used to screen the intestine filters and total cDNA from the gills to screen the gill filters. The cDNA was labelled using $\alpha^{32} \mathrm{P}$-dCTP in a PCR assay. In order not to label the vector, the plasmids containing the cDNA were digested with XhoI/EcoRI to open up the circle. Then M13-20 primer was used in a 'one way' PCR to label the insert. The colonies that hybridised were presumed to be the more abundant ones in the libraries. A total of 3840 non-hybridising clones (the pre- 
sumed rare genes) and 288 hybridising clones were picked from each of the gill and intestine libraries and gridded in duplicate into 384 well microtiterplates. The plates were grown overnight in LB, then glycerol was added and the plates were sealed and stored at $-80^{\circ} \mathrm{C}$.

\section{Enrichment of pBluescript cDNA libraries}

The remaining cDNA was amplified in semi-solid fashion, as described by the manufacturer (Stratagene Cloning Systems, California, USA). Amplification in suspension allowed three-dimensional, uniform growth, which reduced the likelihood of under-representation of particular clones. Up to $5 \times 10^{5} \mathrm{cfu}$ /bottle of primary library was added to each bottle of the semi-solid suspension, before incubation on an ice-water bath for an hour to achieve the required semi-solid state of the medium, followed by incubation for $40-45$ hours at $30^{\circ} \mathrm{C}$. Afterwards the contents of the bottles were spun down and resuspended in $50 \mathrm{ml}$ of 2xLB-glycerol (12.5\%) for the gill library and 100 $\mathrm{ml}$ for the intestine library, ending up with $8 \times 10^{9}$ clones from the gills and $3.1 \times 10^{10}$ clones from the intestine. Total volumes of $1 \mathrm{ml}$ were aliquoted into tubes and subsequently frozen at $-80^{\circ} \mathrm{C}$ for future use.

\section{DNA isolation procedures}

A few microlitres of the pre-screened cDNA library was cultured overnight in LB before the DNA was isolated using the QIAprep 96 Turbo Miniprep kit from QIAGEN in a QIAvac96 according to the manufacturer's manual.

\section{Size determination of the library fractions}

To determine the insert sizes of the clones from the different size fractions, a rough estimate was made for 192 randomly picked clones from each fraction. The insert size was determined by digesting the insert out of the vector using the restriction enzymes EcoRI and XhoI, followed by separation on an agarose gel. The gels were scanned on a Typhoon 9410 Imager and analysed using the ImageQuant TL software, both from Amersham Biosciences.

\section{DNA sequencing}

Sequencing was done from the 5 '-end using T3 as sequencing primer. 5'-sequencing was chosen in order to assign functional annotation to as many transcripts as possible. The sequencing reactions were performed using the ABI PRISM ${ }^{\circledast}$ BigDye $^{\mathrm{TM}}$ Terminators Cycle Sequencing Kit (Applied Biosystems), and run on the ABI 377 (Applied Biosystems) or on the Megabace 1000 (Amersham Pharmacia).

\section{Clustering, sequence comparison and annotation transfer}

Phred [13,14] and cross_match (Green P: unpublished) were used for basecalling and trimming of vector, respectively. The sequences were masked for repeats against a
Danio rerio repeat library [15], and sequences containing contaminants or that were of poor quality were removed. The remaining high quality sequences were assembled and clustered with the Sequencher 4.1 package. In order to examine sequence similarity to known genes, NCBI blast sequence alignment [16] was performed against the NCBI databases [17] (on 08.03.2004). For each sequence, blastx was run against the PDB, Swiss-Prot and nr protein sequence databases, and blastn was run against the $\mathrm{nr}$ nucleotide sequence database. We defined a significant database hit as having an expectation value (E-value) below $1.0 \times 10^{-15}$ for all sequence alignments, except for blastx against PDB where $1.0 \times 10^{-10}$ was used. All sequences that gave a significant blastx hit in Swiss-Prot were annotated by annotation transfer, applying the Gene Ontology (GO) $[18,19]$ assignments for the UniProt database produced by the GOA project of the European Bioinformatics Institute[20]. The gene_association.goa_uniprot database of 26.04.2004 was used [21] together with GO terms from the GO release of 11.05.2004 [22]. The sequences were annotated on the basis of the single best hit in the Swiss-Prot database.

\section{Authors' contributions}

HHL performed the experiments and drafted the manuscript. JKL participated in the sequence comparisons, carried out the annotation transfer, designed the web pages and drafted the bioinformatics section of the manuscript. FP carried out the sequence alignments on the entire SALGENE sequences. AA participated in the sequence comparisons and provided supervision on the bioinformatics section. BH conceived the study, participated in its design and coordination, provided supervision and helped to draft the manuscript. All authors have read and approved the final manuscript.

\section{Acknowledgements}

The authors would like to thank Agate Noer and Kristin Vekterud for their assistance with library construction and sequencing. This work was supported by the EU FAIR Programme SALGENE project (FAIR CT98-43I4); and grant 130162/130 as part of a project entitled "Strategic QTL research plan for disease resistance in Atlantic salmon and cattle" and grant 1396/7/ 140 as part of a project entitled "Salmon Genome Project (SGP)", both supported by the Research Council of Norway.

\section{References}

I. NCBI - National Center for Biotechnology Information [http://www.ncbi.nlm.nih.gov/entrez].

2. Allendorf FWTGH: Tetraploidy and the evolution of salmonid fishes. In Evolutionary genetcs of fishes Edited by: Turner BJ. New York , Plenum Press; 1984: I-53.

3. Ohno S, Wolf U, Atkin NB: Evolution from fish to mammals by gene duplication. Hereditas 1968, 59(I): 169-187.

4. Wolfe KH: Yesterday's polyploids and the mystery of diploidization. Nat Rev Genet 200I, 2(5):333-34I.

5. Boeuf G: Salmonid smolting: a pre-adaptation to the oceanic environment. In Fish Ecophysiology Edited by: Rankin JCJFB. London , Chapman \& Hall; 1993:pp 105-135. 
6. Adams MD, Kelley JM, Gocayne JD, Dubnick M, Polymeropoulos MH, Xiao H, Merril CR, Wu A, Olde B, Moreno RF, et al:: Complementary DNA sequencing: expressed sequence tags and human genome project. Science I99|, 252(50 I3): 165 I-I656.

7. Marra MA, Hillier L, Waterston RH: Expressed sequence tags-ESTablishing bridges between genomes. Trends Genet 1998, I4(I):4-7.

8. Davey GC, Caplice NC, Martin SA, Powell R: A survey of genes in the Atlantic salmon (Salmo salar) as identified by expressed sequence tags. Gene 200I, 263(I-2): $|2|-\mid 30$.

9. Martin SA, Caplice NC, Davey GC, Powell R: EST-based identification of genes expressed in the liver of adult Atlantic salmon (Salmo salar). Biochem Biophys Res Commun 2002, 293(I):578-585.

10. Rise ML, von Schalburg KR, Brown GD, Mawer MA, Devlin RH, Kuipers N, Busby M, Beetz-Sargent M, Alberto R, Gibbs AR, Hunt P, Shukin R, Zeznik JA, Nelson C, Jones SR, Smailus DE, Jones SJ, Schein JE, Marra MA, Butterfield YS, Stott JM, Ng SH, Davidson WS, Koop BF: Development and application of a salmonid EST database and cDNA microarray: data mining and interspecific hybridization characteristics. Genome Res 2004, 14(3):478-490.

II. SGP - Salmon genome project [http://www.salmongenome.no].

12. Chomczynski P, Sacchi N: Single-step method of RNA isolation by acid guanidinium thiocyanate-phenol-chloroform extraction. Anal Biochem 1987, I62(I):156-159.

13. Ewing B, Hillier L, WendI MC, Green P: Base-calling of automated sequencer traces using phred. I. Accuracy assessment. Genome Res 1998, 8(3): 175-185.

14. Ewing B, Green P: Base-calling of automated sequencer traces using phred. II. Error probabilities. Genome Res 1998, 8(3): $186-194$.

15. Repeat Masker [http://ftp.genomewashington.edu/RM/ RepeatMasker.html].

16. Altschul SF, Gish W, Miller W, Myers EW, Lipman DJ: Basic local alignment search tool. J Mol Biol 1990, 215(3):403-4I0.

17. NCBI - National Center for Biotechnology Information [ftp:/ /ftp.ncbi.nih.gov/blast/db].

18. Ashburner M, Ball CA, Blake JA, Botstein D, Butler H, Cherry JM, Davis AP, Dolinski K, Dwight SS, Eppig JT, Harris MA, Hill DP, IsselTarver L, Kasarskis A, Lewis S, Matese JC, Richardson JE, Ringwald M, Rubin GM, Sherlock G: Gene ontology: tool for the unification of biology. The Gene Ontology Consortium. Nat Genet 2000 25(I):25-29.

19. Ashburner M, Lewis S: On ontologies for biologists: the Gene Ontology--untangling the web. Novartis Found Symp 2002, 247:66-80; discussion 80-3, 84-90, 244-52.

20. European Bioinformatics Institute [http://www.ebi.ac.uk GOA].

21. European Bioinformatics Institute [ftp://ftp.ebi.ac.uk/pub/ databases/GO/goa/UNIPROT].

22. The Gene Ontology [http://www.geneontology.org]. .

Publish with Bio Med Central and every scientist can read your work free of charge

"BioMed Central will be the most significant development for disseminating the results of biomedical research in our lifetime. "

Sir Paul Nurse, Cancer Research UK

Your research papers will be:

- available free of charge to the entire biomedical community

- peer reviewed and published immediately upon acceptance

- cited in PubMed and archived on PubMed Central

- yours - you keep the copyright
BioMedcentral 